\title{
Modeling Extensions of Fourier Ptychographic Microscopy
}

\author{
Roarke Horstmeyer ${ }^{1}$, Guoan $\mathrm{Zheng}^{2}$, Xiaoze Ou ${ }^{1}$ and Changhuei Yang ${ }^{1}$ \\ ${ }^{1}$ California Institute of Technology, Department of Electrical Engineering, Pasadena, CA 91125, USA \\ ${ }^{2}$ University of Connecticut Department of Biomedical Engineering, Storrs, CT 06269, USA
}

Fourier ptychographic microscopy (FPM) is a recently introduced procedure that acquires highresolution $\left(0.37^{2} \mu \mathrm{m}^{2}\right)$, wide field of view $\left(120 \mathrm{~mm}^{2}\right)$ giga-pixel optical images [1]. Its operation closely parallels the principles supporting conventional ptychography, the well-known X-ray and electron imaging procedure. Instead of combining a sequence of diffraction images captured from a sample as it is shifted across space, FPM instead images a fixed sample that is illuminated by a series of varied-angle plane waves. As detailed below, while this optical architecture is fundamentally different than conventional ptychography (CP), each setup acquires nearly identical data sequences. In the following, we first mathematically connect FPM and CP, and then use this as a basis to explore how FPM may benefit specific X-ray and electron imaging scenarios.

An FPM system consists of a sample, lens and detector arranged in an imaging configuration with one unique modification: an array of sources placed behind the sample (Fig. 1). As each source is turned on, an image is captured of the sample illuminated with a uniquely angled plane wave. In an optical configuration, the individual sources are light-emitting diodes, and the lens is a wide FOV, low-resolution microscope objective. Following a modified phase retrieval procedure (analogous to iterative procedures used in $\mathrm{CP}$ ), the images may be combined in the Fourier domain to form an estimate of a sample's amplitude and phase, exhibiting approximately 10 times sharper resolution over the objective's fixed FOV (Fig. 1). This wide-field, high-resolution image is similar to CP's final complex reconstructions. However, no mechanical scanning is required, and the demands on the source field's coherence may be relaxed. X-ray and electron CP experiments are commonly challenged by precise movement and source coherence characterization, suggesting FPM may offer a welcome alternative.

We have developed a mathematical model that explicitly connects the operation of FPM and CP. Our model uses a previously utilized phase space function [2], the Wigner distribution function (WDF), to succinctly describe both systems' captured imagery (i.e., data matrix). Previous work has expressed CP's data matrix as a convolution of two Wigner functions: one function that directly maps to a complex description of the sample, and one that maps to the probe field's complex aperture function [2]. Here, we show that FPM's data matrix may likewise be expressed as a convolution of the Wigner function of the sample and the imaging aperture, but each is rotated 90 degrees with respect to those used to model CP. This results in an FPM data matrix that is related to an equivalent CP setup's data matrix by a simple linear transformation [3].

The importance of a linear relationship between CP and FPM is two-fold. First, by casting FPM within the framework of CP, we can directly compare the performance of each system, even though their imaging architectures are completely distinct. Comparing tolerances to various sources of noise, misalignment, optic aberrations and other imperfections yields a tradeoff space that highlights the specific benefits of each procedure. For example, most electron microscopes include highly precise illumination-steering magnets that enable dark-field capture. Microscopes with an imprecise mechanical sample translation stage may instead adopt FPM's illumination steering and subsequent recovery. This helps minimize experimental instabilities during thin-sample imaging. Alternatively, X-ray 
ptychography setups similarly limited by sample translation instability may instead add a rotatable selective Bragg grating in a fixed sample's optical path. The grating will relay different sample spectrum regions to the detector, again enabling FPM-based recovery. Such alternative configurations are an ongoing area of investigation.

A second benefit of our framework connecting CP and FPM is its ability to completely describe partial coherence effects (Fig. 2). Little work to date has detailed how partial coherence impacts either CP or FPM reconstructions. Our model enables us to compare how the performance of each procedure degrades with reduced source coherence. This additional piece of information will allow future postprocessing methods to account for source statistics, improve solution accuracy and identify scenarios where increasingly incoherent sources may still yield acceptable phase recovery.

In conclusion, our proposed mathematical model formally joins an imaging technique that has found success in the optical regime with well-established principles from ptychography. We hope this merger will facilitate new insights at the intersection of these two computation-based imaging procedures.

\section{References:}

[1] G. Zheng, R. Horstmeyer, and C. Yang, Nature Photon. 7 (2013), p. 739-745.

[2] J. M. Rodenburg, and R. H. T. Bates, Phil. Trans. R. Soc. Lond. A 339 (1992), p. 521-553.

[3] R. Horstmeyer and C. Yang, Opt. Express 22(1) (2014), p. 338-358.
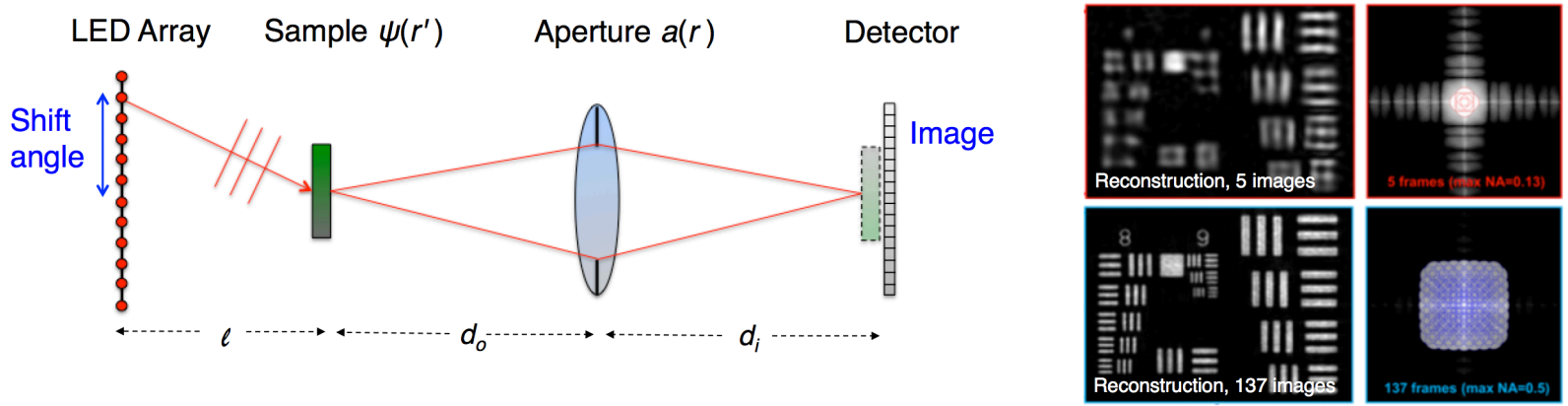

Figure 1: The FPM setup and associated recovery results in $x$-space and $k$-space. After processing all 137 images, AF Group $9(0.78 \mu \mathrm{m})$ is clearly resolved by our $2 \mathrm{X}$ objective aperture $(5.5 \mu \mathrm{m}$ resolution $)$.

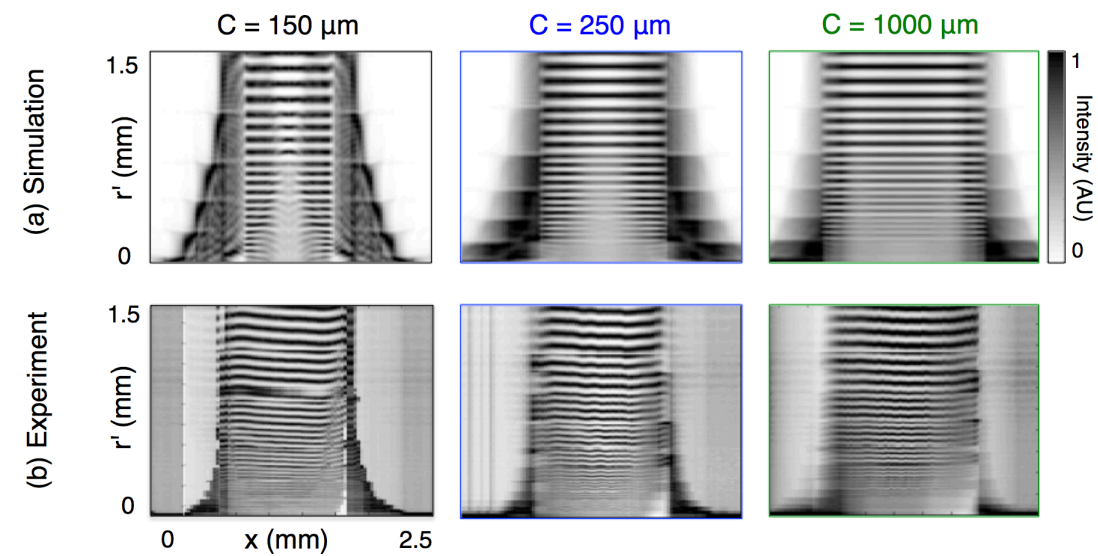

(c) Grating profile

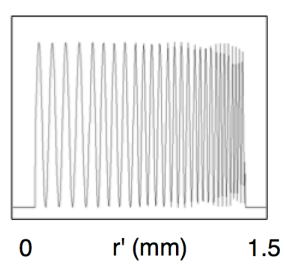

(d) Source profiles

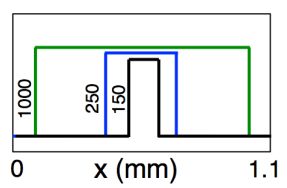

Figure 2: (a) Simulation and (b) experimental FPM data matrices for a $1 \mathrm{D}$ chirped grating verify the validity of our proposed mathematical model. As the size of the incoherent illumination source $c$ is increased, each data matrix is extended and blurred along the angular shift variable $x$, as predicted by partial coherence theory. 